\title{
Compact Ultrawideband Mimo Antenna for Mobile Applications
}

\author{
Sinu Mathew $\mathrm{K}^{1}$, R Sathyabama ${ }^{2}$ \\ ${ }^{1}$ Hindusthan college of engineering and technology Coimbatore) \\ ${ }^{2}$ Assistant Professor Hindusthan college of engineering and technology Coimbatore)
}

\begin{abstract}
A new broadband multiple-input multiple-output (MIMO) antenna with good isolation and compact size is proposed. The proposed antenna consists of two G-shaped elements in the upper layer and two inverted $L$ protrude branches and a $T$ slot etched in ground which is used to reduce the mutual coupling. This planar antenna has a bandwidth of $100 \%$ with $\left|S_{11}\right| \leq-10 \mathrm{~dB}$ from $2.26 \mathrm{GHz}$ to $6.78 \mathrm{GHz}$. The value of isolation between the two antenna elements is more than $22.5 \mathrm{~dB}$ in the whole band. The experimental results verify the simulations. This project presents an ultra-wideband MIMO antenna covering WCDMA, WLAN, WiMax and UWB bands for mobile handset applications. The designed antenna consists of a folded monopole antenna coupled with inverted-L element and meander lines. The proposed antennas are symmetrically placed on top part of the mobile handset ground for MIMO antenna technology. Meander lines are positioned between two antennas for improving the isolation in operation bands. The size of the designed MIMO antenna is $55 \mathrm{~mm} \times$ 13:5 mm. The isolation between two antennas is higher than $17.2 \mathrm{~dB}$. Due to the compact size and ultra-wide bandwidth, it is good for a mobile handset antenna that has a limited space available.
\end{abstract}

\section{Multiple-Input-Multiple-Output(Mimo) Antenna}

\section{Introduction}

MIMO technology has been reported for several years, which significantly enhances the performance of wireless communication systems. One important requirement for MIMO antenna systems is the need for good isolation between antenna elements. In some applications, such as cellular communication, MIMO technology is easy to be implemented at the base station where antenna elements separate many wavelengths, and good isolation is easily available. However, in mobile terminals, reaching high isolation in small size is difficult to fulfil. Various methods have been devised to improve isolation between the elements of a narrowband MIMO antenna. Good isolation is achieved by adding parasitic elements, which can create a reverse coupling to reduce mutual coupling. However, the method of adding parasitic elements is sensitive to the position of the parasitic elements.

In addition, the use of electromagnetic band-gap (EBG) is able to suppress surface wave propagation and thus improve the isolation between radiating elements. However this method occupies significant areas. Other isolation enhanced techniques, such as incorporating a protruded ground between the antennas, inserting slits into the ground, applying multi-port conjugate (MC) match, are also used.

\section{Mobile Device}

A mobile device (also known as a handheld computer or simply handheld) is a small, handheld computing device, typically having a display screen with touch input and/or a miniature keyboard and weighing less than 2pounds $(0.91 \mathrm{~kg})$. Apple, $\underline{\text { Nokia }}, \underline{\mathrm{HTC}}, \underline{\mathrm{LG}}, \underline{\text { BlackBerry }}$, and Motorola Mobility are just a few examples of the many manufacturers that produce these types of devices. A handheld computing device has an operating system (OS), and can run various types of application software, known as apps. Most handheld devices can also be equipped with Wi-Fi, Bluetooth, and GPS capabilities that can allow connections to the Internet and other Bluetooth-capable devices, such as an automobile or a microphone headset. A camera or media player feature for video or music files can also be typically found on these devices along with a stable battery power source such as a lithium battery.

\section{Multiple-Input Multiple-Output (MIMO)}

MIMO (Multiple Input, Multiple Output) is an antenna technology for wireless communications in which multiple antennas are used at both the source (transmitter) and the destination (receiver). The antennas at each end of the communications circuit are combined to minimize errors and optimize data speed. All wireless products with 802.11n support MIMO, which is part of the technology that allows $802.11 \mathrm{n}$ to reach much higher speeds than products without $802.11 \mathrm{n}$. MIMO technology takes advantage of a natural radio-wave phenomenon called multipath. With multipath, transmitted information bounces off walls, ceilings, and other objects, reaching the receiving antenna multiple times via different angles and at slightly different times. Which dramatically increase performance and range. 

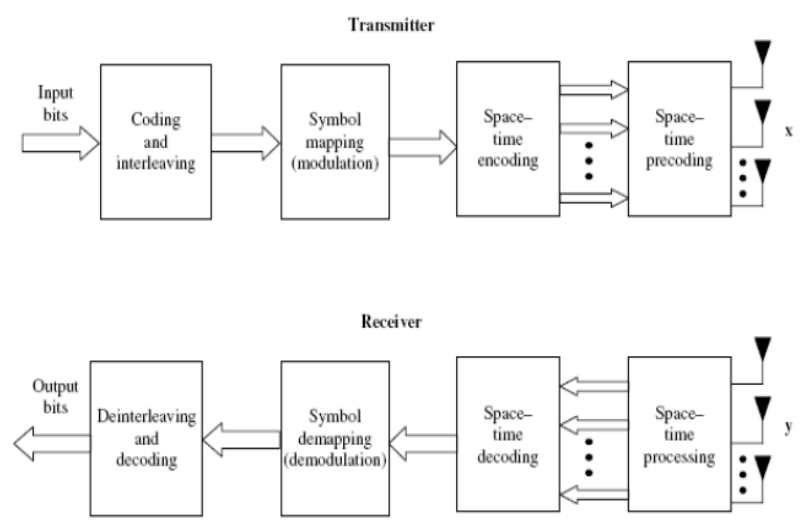

Fig 1 A typical MIMO system including the signal processing subsystem

\section{Antenna Designe}

Two ultrawideband antennas are symmetrically arrayed, and parasitic meander lines are located between them on the substrate to improve the isolation. The proposed antenna is shown in Fig. 2.(b). The previously designed ultrawideband antenna consists of a printed folded monopole antenna coupled with a parasitic inverted-L element. This antenna cannot cover the WCDMA band. Therefore, the length of the inverted-L element was lengthened to cover the WCDMA band, and the gap between the folded monopole and the inverted-L element was changed for impedance matching in this study. In addition, an open stub is inserted in the printed folded monopole to reject the WLAN $(5.15 \mathrm{GHz}-5.85 \mathrm{GHz})$ band, which interferes with the UWB band, while maintaining the size of the antenna. The overall length of the open stub from the feed point is $17 \mathrm{~mm}$. This is determined to be approximately $0.5 \lambda$ of $5.5 \mathrm{GHz}$ because it has high input impedance by operating $\lambda / 2$ open circuit.

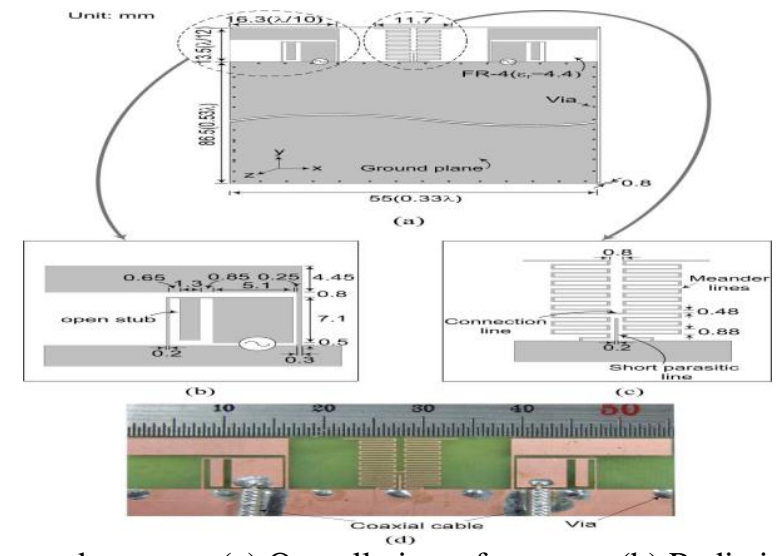

Figure : Structure of the proposed antenna. (a) Overall view of antenna. (b) Radiation element on left-hand side. (c)Meander lines for improving isolation.

(d) Fabricated prototype antenna

\section{Simulated And Measured Results}

The simulated and measured S-parameters are shown in Fig. 2.(a) and (b). CST Microwave Studio 2010 was used for the simulation. The two results are in good agreement. The input impedance was well matched, and WLAN band-rejection was observed. The 10-dB bandwidth was measured as $1.85-11.9 \mathrm{GHz}$ (6.43:1), except for the rejected WLAN band, which is $700 \mathrm{MHz}(5.15-5.85 \mathrm{GHz}$, above 10-dB return loss). This antenna covers the WCDMA (1.92-2.17 GHz), WiMAX (2.3, $2.5 \mathrm{GHz})$, WLAN (2.4 GHz), and UWB $(3.1-10.6 \mathrm{GHz})$ bands. $\mathrm{S}_{21}$ of the antennas was measured to be lower than $-17.2 \mathrm{~dB}$ in $1.92-10.6 \mathrm{GHz}$.

\section{Microstrip Antennas - Introduction}

\section{Implementation Phase}

In high-performance aircraft, spacecraft, satellite, and missile applications, where size, weight, cost, performance, ease of installation, and aerodynamic profile are constraints, low - profile antennas may be required. Presently there are many other government and commercial applications, such as mobile radio and wireless communications that have similar specifications. To meet these requirements, microstrip antennas can be used. These antennas are low profile, conformable to planar and nonplanar surfaces, simple and inexpensive 
to manufacture using modern printed-circuit technology, mechanically robust when mounted on rigid surfaces, compatible with MMIC designs, and when the particular patch shape and mode are selected, they are very versatile in terms of resonant frequency, polarization, pattern, and impedance. In addition, by adding loads between the patch and the ground plane, such as pins and varactor diodes, adaptive elements with variable resonant frequency, impedance, polarization, and pattern can be designed.

\section{Basic Characterestics}

Microstrip antennas received considerable attention starting in the 1970s, although the idea of a microstrip antenna can be traced to 1953 and a patent in 1955. Microstrip antennas, as shown in Figure 5.1(a), consist of a very thin ( $t<<\lambda_{0}$, where $\lambda_{0}$ is the free-space wavelength) metallic strip (patch) placed a small fraction of a wavelength ( $h \ll \lambda_{0}$, usually $\left.0.003 \lambda_{0} \leq h \leq 0.05 \lambda_{0}\right)$ above a ground plane. The microstrip patch is designed so its pattern maximum is normal to the patch (broadside radiator). This is accomplished by properly choosing the mode (field configuration) of excitation beneath the patch. End-fire radiation can also be accomplished by judicious mode selection. For a rectangular patch, the length $L$ of the element is usually $\lambda_{0} / 3<$ $L<\lambda_{0} / 2$. The strip (patch) and the ground plane are separated by a dielectric sheet (referred to as the substrate), as shown in Figure 5.(a).

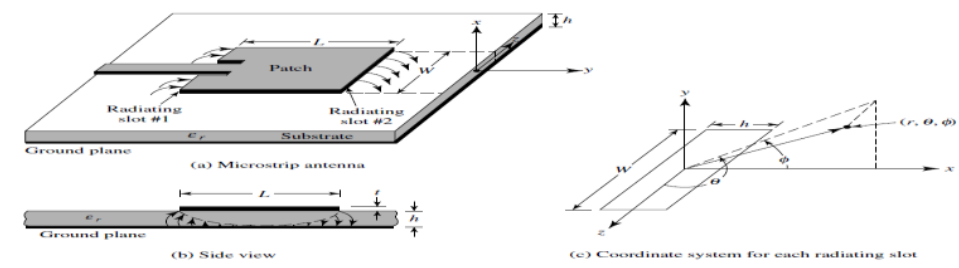

Figure 3: Microstrip antenna and coordinate system

\section{Antenna Design}

Based on the simplified formulation that has been described, a design procedure is outlined which leads to practical designs of rectangular microstrip antennas. The procedure assumes that the specified information includes the dielectric constant of the substrate $\left(\varepsilon_{r}\right)$, the resonant frequency $\left(f_{r}\right)$, and the height of the substrate $h$. The procedure is as follows:

A. Specify: $\varepsilon_{\mathrm{r}}, \mathrm{f}_{\mathrm{r}}($ in $\mathrm{Hz}$ ), and $\mathrm{h}$

B. Determine: $\mathrm{W}, \mathrm{L}$

C. Design procedure:

1. For an efficient radiator, a practical width that leads to good radiation efficiencies

$$
\boldsymbol{W}=\frac{1}{2 f_{r} \sqrt{\mu_{\mathrm{O}} \epsilon_{\mathrm{O}}}} \sqrt{\frac{2}{\epsilon_{r}+1}}=\frac{v_{\mathrm{o}}}{2 f_{r}} \sqrt{\frac{2}{\epsilon_{r}+1}}
$$

Where $v_{0}$ is the free-space velocity of light.

2. Determine the effective dielectric constant of the microstrip antenna using (4-1).

3. Once $W$ is found using (4-4), determine the extension of the length $\Delta L$ using

(4-2).

4. The actual length of the patch can now be determined by

$$
L=\frac{1}{2 f_{r} \sqrt{\epsilon_{\text {reff }}} \sqrt{\mu_{\mathrm{O}} \epsilon_{\mathrm{O}}}}-2 \Delta L
$$

\footnotetext{
Design Specifications Of Proposed Antenna

- Frequency of operation ( $\mathrm{fo}=15 \mathrm{GHz}$ )

- Dielectric constant of the substrate ( $\mathrm{gr}=4.4$ for FR-4 substrate )

- Height of dielectric substrate $(\mathrm{h}=0.8 \mathrm{~mm})$

- $\quad \mathrm{BW}=1.85 \mathrm{GHz}-11.9 \mathrm{GHz}$

- Size of the Antenna $=55^{*} 13.5 \mathrm{~mm}^{\wedge} 2$

- $\quad$ Size of the Isolation $=11.7 * 13.5 \mathrm{~mm}^{\wedge} 2$

- $\mathrm{ECC}=$ less than 0.18 (for $1.92 \mathrm{GHz}-10.6 \mathrm{GHz}$ )
} 
Return Loss, RL(dB)= $10 \log \mathrm{Pi} / \mathrm{Pr}$

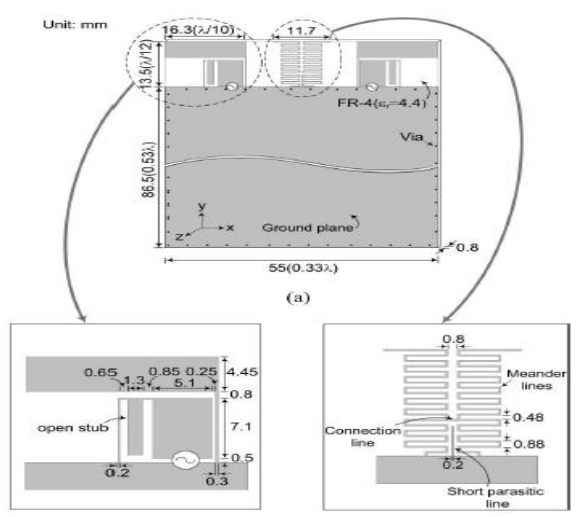

Figure 4 Designed Measurements of the Antenna

Simulation Usnig Cst Microwave Studio

III. Design Environment

CST MICROWAVE STUDIO (CST MWS) is a powerful tool for the 3D electromagnetic (EM) simulation of high frequency components. CST MWS offers unparalleled performance, making it first choice in technology leading R\&D departments.CST MWS enables the fast and accurate analysis of high frequency (HF) devices such as antennas, filters, couplers, planar and multi-layer structures and SI and EMC effects. Exceptionally user friendly, CST MWS quickly gives you an insight into the EM behavior of your high frequency designs.

\section{Choice of Solver Modules}

Users of CST MWS can choose between six powerful solver modules each offering distinct advantages in their own domains. In the CST Complete Technology approach, the seamless integration of these solvers in one user interface enables the easy selection of the most appropriate solver for a given problem class, delivering improved simulation performance and unprecedented simulation reliability through cross verification.

\section{CST Simulation Acceleration}

Accurate conformal methods improve simulation performance by orders of magnitude compared to standard approaches. Additionally, efficient implementation on powerful hardware delivers a competitive edge. CST offers a multitude of simulation acceleration options such as multi-CPI processing, GPU processing, cluster computing, and distributed computing.

Inputs To The Ports

\section{Simulation And Results}

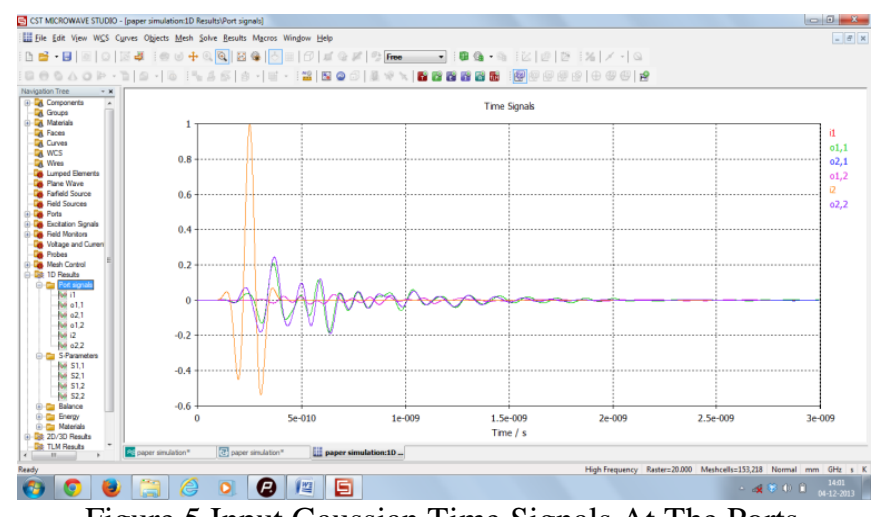

Figure 5 Input Gaussian Time Signals At The Ports 


\section{Radiation Patterns}

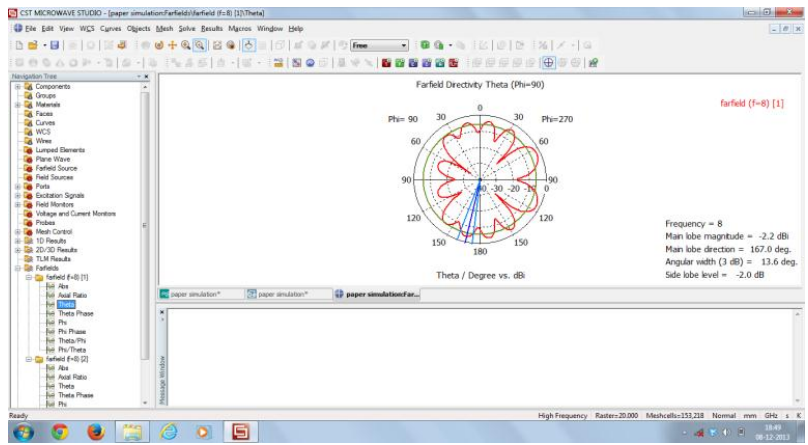

Figure 6 Simulated Radiation Pattern 1

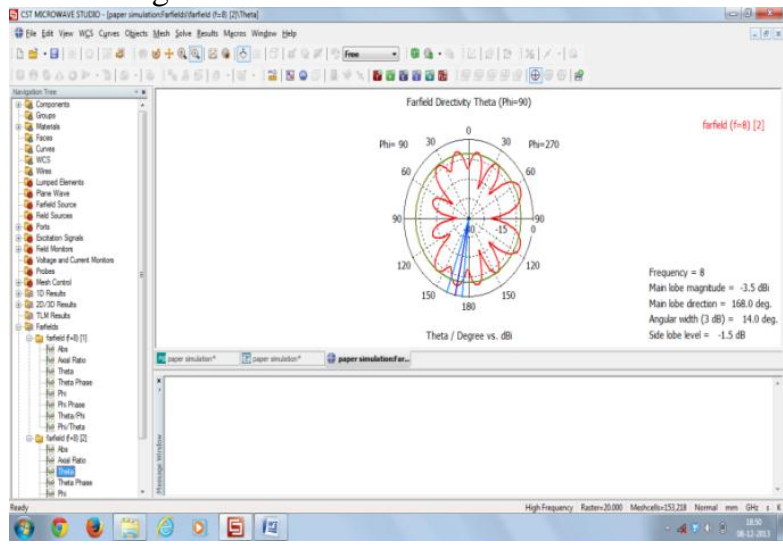

Figure 7 Simulated Radiation Pattern 2

\section{S-Parameter Analysis Results}

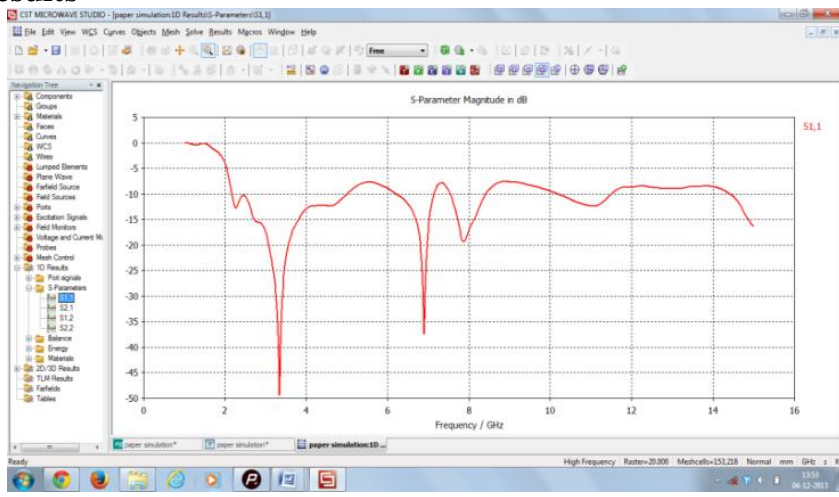

Figure $8 \mathrm{~S}_{11}$ Characteristics of the Antenna

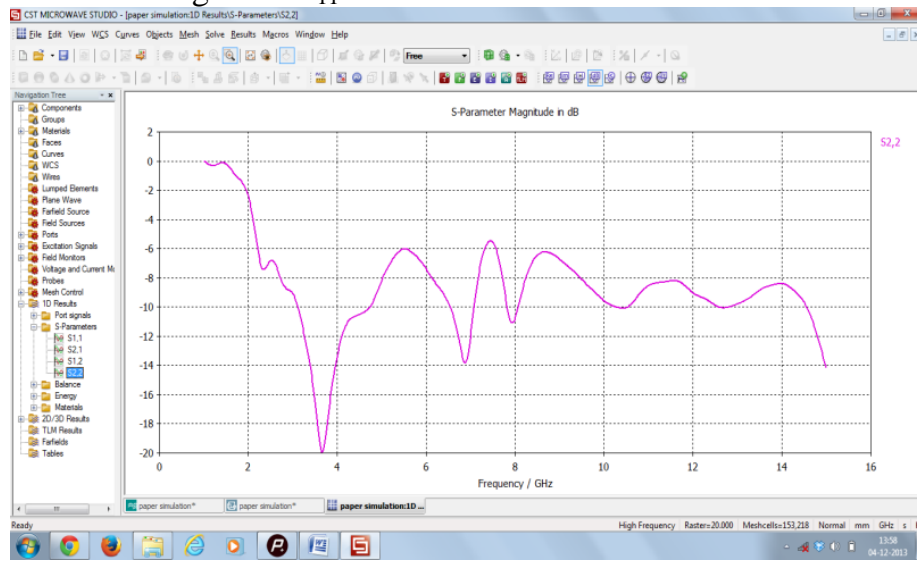

Figure $9 \mathrm{~S}_{22}$ Characteristics of the Antenna 


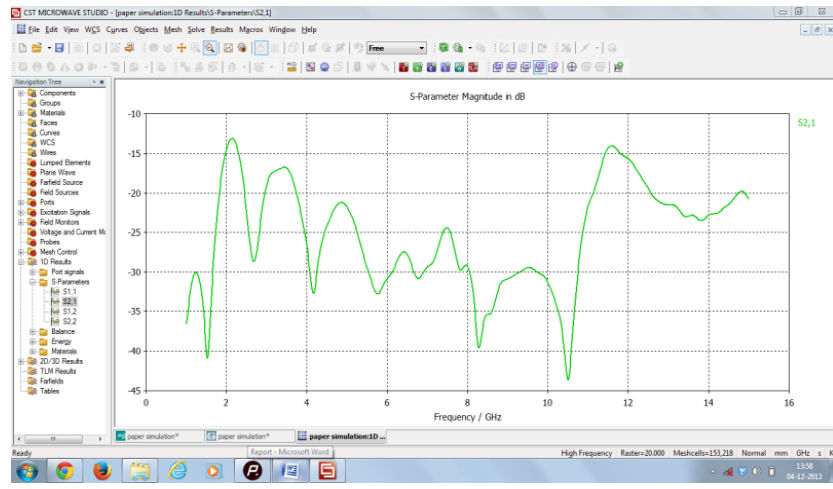

Figure $10 \mathrm{~S}_{21}$ Characteristics of the Antenna

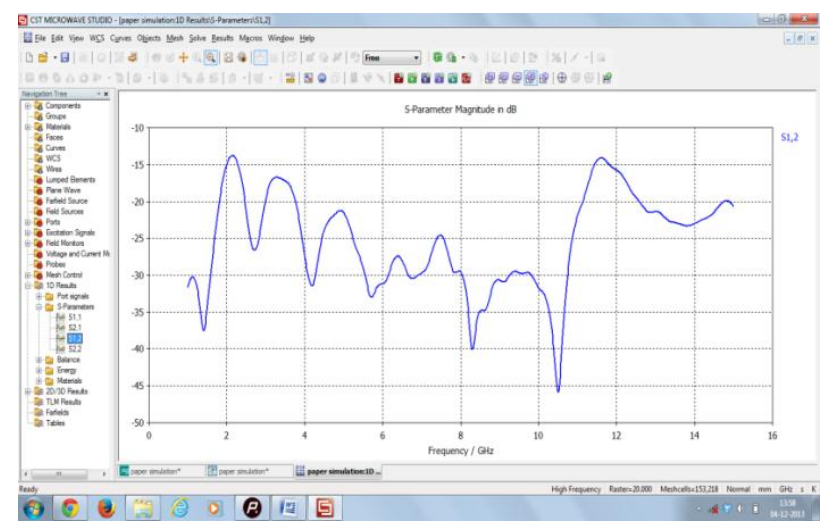

Figure $11 \mathrm{~S}_{12}$ Characteristics of the Antenna

\section{Inference}

\section{Conclusion}

This project was successfully simulated with CST Microwave Studio with the design technology of microstrip rectangular patch antenna. The antenna was designed to cover WCDMA (1.92-2.17 GHz), WiMAX (2.3 GHz, $2.5 \mathrm{GHz})$, WLAN $(2.4 \mathrm{GHz})$, and UWB $(3.1-10.6 \mathrm{GHz})$ band for mobile data communication. The antenna was designed to provide low attenuation with low return loss. The S-parameter analysis for individual antennas and the mutual coupling of both the antennas has been obtained.

\section{Scope For The Future Work}

Proposed work is designing antenna which can cover all the ultrawideband frequencies with a parasitic inverted-L element which is coupled with printed monopole antenna. This antenna may enhance the band width of the present design and which will also reject the WLAN band of 5.15-5.85 GHz which may interfere the ultrawideband for data communication. An open stub is inserted in the folded monopole to block $5 \mathrm{GHz}$ WLAN band.

\section{References}

[1] A.Najam, Y. Duroc, and S. Tedjni, "UWB-MIMO antenna with novel stub structure," Prog. Electromagn. Res. C, vol. 19, pp. 245257, Feb. 2011.

[2] Ayatollahi, M., Q. Rao, and D. Wang, "A compact, high isolation and wide bandwidth antenna array for long term evolution wireless devices," IEEE Trans. on Antennas and Propag., Vol. 45, 601 \{602, 2012.

[3] G. J. Foschini, "On limits of wireless communications in a fading environment when using multiple antennas," Wireless Pers. Commun., vol.6, no. 3, pp. 311-335, 1998.

[4] J.-F Li, Q.-X. Chu, and T.-G. Huang, "A compact wideband MIMO antenna with two novel slits," IEEE Trans. Antennas Propag., vol. 60, no. 2, pp. 482-489, Feb. 2012.

[5] Jae-Min Lee, Ki-Baek Kim, Hong-Kyun Ryu, and Jong-Myung Woo, "A Compact Ultrawideband MIMO Antenna With WLAN Band-Rejected operation for Mobile Devices" IEEE ANTENNAS AND WIRELESS PROPAGATION LETTERS, VOL. 11, 2012

[6] K.-B. Kim, H.-K. Ryu, and J.-M. Woo, "Compact wideband folded monopole antenna coupled with parasitic inverted-L element for laptop computer applications," Electron. Lett., vol. 47, no. 5, pp. 301-303, Mar. 2011.

[7] Li, Z., Z. Du, M. Takahashi, K. Saito, and K. Ito, IReducing mutual coupling of MIMO antennas with parasitic elements for mobile terminals," IEEE Trans. on Antennas and Propag., Vol. 60, 473\{481, 2012. 